CRM-1846 (1993)

\title{
Hamiltonian Structure of Equations Appearing in Random Matrices ${ }^{\dagger}$
}

\author{
J. Harnad ${ }^{1}$ \\ Department of Mathematics and Statistics, Concordia University \\ 7141 Sherbrooke W., Montréal, Canada H4B 1R6, and \\ Centre de recherches mathématiques, Université de Montréal \\ C. P. 6128-A, Montréal, Canada H3C 3J7 \\ C. A. Tracy $^{2}$ \\ Department of Mathematics and Institute of Theoretical Dynamics \\ University of California, Davis, CA 95616, USA \\ H. Widom ${ }^{3}$ \\ Department of Mathematics \\ University of California, Santa Cruz, CA 95064, USA.
}

\begin{abstract}
The level spacing distributions in the Gaussian Unitary Ensemble, both in the "bulk of the spectrum," given by the Fredholm determinant of the operator with the sine kernel $\frac{\sin \pi(x-y)}{\pi(x-y)}$ and on the "edge of the spectrum," given by the Airy kernel $\frac{A i(x) A i^{\prime}(y)-A i(y) A i^{\prime}(x)}{(x-y)}$, are determined by compatible systems of nonautonomous Hamiltonian equations. These may be viewed as special cases of isomonodromic deformation equations for first order $2 \times 2$ matrix differential operators with regular singularities at finite points and irregular ones of Riemann index 1 or 2 at $\infty$. Their Hamiltonian structure is explained within the classical $R$-matrix framework as the equations induced by spectral invariants on the loop algebra $\widetilde{s l}(2)$, restricted to a Poisson subspace of its dual space $\widetilde{s l}_{R}^{*}(2)$, consisting of elements that are rational in the loop parameter.
\end{abstract}

$\dagger$ Research supported in part by the Natural Sciences and Engineering Research Council of Canada and by the National Science Foundation, U.S.A., DMS-9001794 and DMS-9216203.

1 e-mail address: harnad@alcor.concordia.ca or harnad@mathcn.umontreal.ca

2 e-mail address: catracy@ucdavis.edu

3 e-mail address:widom@cats.ucsc.edu 


\section{Introduction}

It has long been known for a class of exactly solvable models in statistical mechanics that the correlation functions are expressible in terms of Painlevé transcendents or more generally solutions to completely integrable differential equations possessing the Painlevé property [WMTB], [MTW], [SMJ]. (For a review see [Mc].) It was the great insight of the Kyoto School to invent the notion of a $\tau$-function associated to a system of completely integrable differential equations $[\mathbf{S M J}],[\mathbf{J M M S}],[\mathbf{J M U}],[\mathbf{J M}]$ and to realize that in this class of solvable models the correlation functions are $\tau$-functions.

In the theory of random matrices it has also long been known (see, e.g., [Po], [Me1]) that the level spacing distributions are expressible in terms of the Fredholm determinant of an integral operator. In a particular scaling limit the kernel of this integral operator is the sine kernel. For this case it was shown in [JMMS] that the Fredholm determinant was also a $\tau$-function. This then leads in the simplest case to formulas for the level spacing distributions in terms of a particular Painlevé V transcendent. More generally, the deformation equations consist of a compatible set of nonautonomous Hamiltonian equations.

Since the original work of [JMMS] on random matrices and impenetrable bosons, the methods for deducing the relevant deformation equations and properties of the $\tau$-functions have been simplified and generalized, both in the study of random matrices [BTW], [Me2], [Dy], [MM], [TW1], [TW2], [W] and quantum correlation functions [IIKS], [IIKV]. The Hamiltonian structure of the underlying deformation equations can most simply be understood as a nonautonomous version of the equations induced by spectral invariants on a loop algebra with respect to a suitably chosen classical $R$-matrix Poisson bracket structure $[\mathbf{H}]$.

In the following section, the problem of computing level spacing distributions for the Gaussian Unitary Ensemble (GUE), both in the "bulk of the spectrum" and at the "edge of the spectrum" is formulated in these terms - the two cases differing only in the kernels resulting from the different scaling limits. For the first case, this is the above mentioned sine kernel

$$
\frac{1}{\pi} \frac{\sin \pi(x-y)}{x-y},
$$

while for the second it is the Airy kernel

$$
\frac{\operatorname{Ai}(\mathrm{x}) \operatorname{Ai}^{\prime}(\mathrm{y})-\operatorname{Ai}(\mathrm{y}) \operatorname{Ai}^{\prime}(\mathrm{x})}{\mathrm{x}-\mathrm{y}} .
$$

In $\S 2$, the resulting Hamiltonian equations are explained in terms of spectral invariants on the dual space $\widetilde{s l}^{*}(2)$ of the loop algebra $\widetilde{s l}(2)$, with respect to a standard classical $R$-matrix 
Poisson bracket structure, restricted to Poisson subspaces consisting of rational coadjoint orbits.

\section{Spectral Distributions for Random Matrices}

\section{1a. Fredholm Determinant Representation}

The GUE measure on the spectral space of $N \times N$ hermitian matrices with eigenvalues $\left(X_{1}, \ldots, X_{N}\right)$ is $[\mathrm{Me1}]$

$$
P_{N}\left(X_{1}, \ldots, X_{N}\right) d X_{1} \cdots d X_{N}=C_{N} \prod_{j<k}\left|X_{j}-X_{k}\right|^{2} \exp \left(-\sum_{j} X_{j}^{2}\right) d X_{1} \cdots d X_{N}
$$

Averaging over all but $n$ of the eigenvalues gives the $n$-point correlation function [Me1]:

$$
\begin{aligned}
R_{n, N}\left(X_{1}, \ldots, X_{n}\right) & =\frac{N !}{(N-n) !} \int_{-\infty}^{\infty} \cdots \int_{-\infty}^{\infty} P_{N}\left(X_{1}, \ldots, X_{N}\right) d X_{n+1} \cdots d X_{N} \\
& =\operatorname{det}\left(\left.K_{N}\left(X_{j}, X_{k}\right)\right|_{j, k=1} ^{n}\right)
\end{aligned}
$$

where

$$
K_{N}(X, Y):=\sum_{k=0}^{N-1} \varphi_{k}(X) \varphi_{k}(Y)
$$

Here $\left\{\varphi_{k}(X)\right\}$ is the sequence obtained by orthonormalizing the sequence $\left\{X^{k} \exp \left(-X^{2} / 2\right)\right\}$ over $(-\infty, \infty)$. In particular $R_{1, N}(X)$, the density of eigenvalues at $X$, equals $K_{N}(X, X)$.

For fixed $X$,

$$
R_{1, N}(X) \sim \frac{1}{\pi} \sqrt{2 N} \text { as } N \rightarrow \infty .
$$

The scaling limit in the bulk of the spectrum at the point $X_{0}$ is the limit

$$
N \rightarrow \infty, X_{j} \rightarrow X_{0} \text {, such that } x_{j}=\left(X_{j}-X_{0}\right) R_{1, N}\left(X_{0}\right) \text { is fixed. }
$$

In this limit the scaled $\mathrm{n}$-point correlation functions $R_{n}\left(x_{1}, \ldots, x_{n}\right)$, defined by

$$
R_{n}\left(x_{1}, \ldots, x_{n}\right) d x_{1} \cdots d x_{n}=\lim _{\substack{N \rightarrow \infty, X_{j} \rightarrow X_{0} \\ x_{j} \text { fixed }}} R_{n, N}\left(X_{1}, \ldots, X_{n}\right) d X_{1} \cdots d X_{n}
$$

are given by $[\mathbf{M e 1}]$

$$
R_{n}\left(x_{1}, \ldots, x_{n}\right)=\operatorname{det}\left(\left.K\left(x_{j}, x_{k}\right)\right|_{j, k=1} ^{n}\right)
$$


where

$$
K(x, y):=\frac{1}{\pi} \frac{\sin \pi(x-y)}{x-y}
$$

Now consider a set of "spectral windows"

$$
J=J_{1} \cup \cdots \cup J_{m}
$$

where

$$
\left\{J_{k}=\left[a_{2 k-1}, a_{2 k}\right] \subset \mathbf{R}\right\}_{k=1, \ldots m}, \quad a_{1}<a_{2}<\ldots<a_{2 m}
$$

is an ordered set of disjoint intervals on the real axis. The probability that a randomly chosen GUE matrix has precisely $\left\{n_{1}, n_{2}, \ldots, n_{m}\right\}$ scaled eigenvalues in the respective intervals $\left\{J_{1}, J_{2}, \ldots, J_{m}\right\}$ is given by the level spacing distribution:

$$
E\left(n_{1}, \ldots, n_{m}, J\right)=\left.\frac{(-1)^{n}}{n_{1} ! \cdots n_{m} !} \frac{\partial^{n} D(J ; \lambda)}{\partial \lambda_{1}^{n_{1}} \cdots \partial \lambda_{m}^{n_{m}}}\right|_{\lambda_{1}=\cdots=\lambda_{m}=1}
$$

with $n=n_{1}+\cdots+n_{m}$. Here $D(J ; \lambda)$ is the Fredholm determinant

$$
D(J ; \lambda):=\operatorname{det}(I-\widehat{K})
$$

of the integral operator $\widehat{K}$ with kernel

$$
K_{J}(x, y):=\sum_{k=1}^{m} \lambda_{k} K(x, y) \chi_{J_{k}}(y)
$$

where $\chi_{J_{k}}(y)$ is the characteristic function for the interval $J_{k}$, and

$$
\lambda:=\left(\lambda_{1}, \lambda_{2}, \ldots, \lambda_{m}\right)
$$

denotes the set of generating function parameters. The case of one interval is in [Me1]. The more general formula (1.10) was derived in [TW1].

In the following, we shall see that $D(J ; \lambda)$ is determined by a system of partial differential equations consisting of a compatible set of nonautonomous Hamiltonian equations defined on a suitable phase space. The quantity of central interest will be the total differential

$$
\omega(a):=d_{a} \log D(J ; \lambda)=\sum_{i=1}^{2 m} \frac{\partial \log D(J ; \lambda)}{\partial a_{i}} d a_{i}
$$

of the logarithm of the generating function $D(J ; \lambda)$, taken with respect to the set of parameters

$$
a:=\left(a_{1}, \ldots, a_{2 m}\right)
$$


giving the boundaries of the spectral domains. The dynamical equations of Jimbo, Miwa, Môri and Sato [JMMS], which determine the dependence of this quantity on the parameters $\left(a_{1}, \ldots, a_{2 m}\right)$, are given below, following the approach developed in [TW1]. We note that in [JMMS], [TW1], and [TW2] the case of equal $\lambda_{j}$ 's only was considered in the derivation of the equations.

\section{1b. Dynamics of Distribution Functions}

The key object in what follows is the resolvent operator

$$
\widehat{R}:=(1-\widehat{K})^{-1} \widehat{K}
$$

Introducing the functions

$$
\begin{aligned}
& Q(x, a):=(1-\widehat{K})^{-1} A \\
& P(x, a):=(1-\widehat{K})^{-1} A^{\prime},
\end{aligned}
$$

where

$$
A(x):=\frac{1}{\pi} \sin \pi x
$$

the kernel of the resolvent operator can be shown to be given by

$$
\begin{aligned}
& R(x, y)=\sum_{k=1}^{m} \lambda_{k} \frac{Q(x, a) P(y, a)-P(x, a) Q(y, a)}{x-y} \chi_{J_{k}}(y), \quad x \neq y, \\
& R(x, x)=\sum_{k=1}^{m} \lambda_{k}\left(\frac{d Q}{d x}(x, a) P(x, a)-\frac{d P}{d x}(x, a) Q(x, a)\right) \chi_{J_{k}}(x) .
\end{aligned}
$$

Using the identity

$$
d_{a} \log (\operatorname{det}(I-\widehat{K}))=-\operatorname{tr}\left((I-\widehat{K})^{-1} d_{a} \widehat{K}\right)
$$

it is easy to show that

$$
\omega(a)=\sum_{j=1}^{2 m}(-1)^{j+1} R\left(a_{j}, a_{j}\right) d a_{j},
$$

where $R\left(a_{j}, a_{k}\right)$ is obtained by taking the limits $(x, y) \rightarrow\left(a_{j}, a_{k}\right)$ inside $J$. Let

$$
\begin{aligned}
q_{j} & :=\lim _{\substack{x \rightarrow a_{j} \\
x \in J}} \sqrt{\lambda_{j}} Q(x, a) \\
p_{j} & :=\lim _{\substack{x \rightarrow a_{j} \\
x \in J}} \sqrt{\lambda_{j}} P(x, a), \quad j=1, \ldots, 2 m .
\end{aligned}
$$


Then we have

$$
\begin{aligned}
& R\left(a_{j}, a_{k}\right)=\frac{q_{j} p_{k}-p_{j} q_{k}}{a_{j}-a_{k}}, \quad j \neq k \\
& R\left(a_{j}, a_{j}\right)=\pi^{2} q_{j}^{2}+p_{j}^{2}+\sum_{k=1}^{2 m}(-1)^{k} R\left(a_{j}, a_{k}\right) R\left(a_{k}, a_{j}\right)\left(a_{j}-a_{k}\right) .
\end{aligned}
$$

Thus, the parameter dependence of $\operatorname{det}(I-\widehat{K})$ is determined through the quantities $\left\{q_{j}, p_{j}\right\}_{j=1, \ldots 2 m}$. Differentiating equations $(1.17 \mathrm{a}, \mathrm{b})$ with respect to the parameters $a_{j}$ and taking the appropriate limits, these may be shown to satisfy the following system of equations:

$$
\begin{aligned}
& \frac{\partial q_{j}}{\partial a_{k}}=(-1)^{k} R\left(a_{j}, a_{k}\right) q_{k}, \quad j \neq k \\
& \frac{\partial p_{j}}{\partial a_{k}}=(-1)^{k} R\left(a_{j}, a_{k}\right) p_{k}, \quad j \neq k \\
& \frac{\partial q_{j}}{\partial a_{j}}=p_{j}-\sum_{k \neq j}(-1)^{k} R\left(a_{j}, a_{k}\right) q_{k} \\
& \frac{\partial p_{j}}{\partial a_{j}}=-\pi^{2} q_{j}-\sum_{k \neq j}(-1)^{k} R\left(a_{j}, a_{k}\right) p_{k} .
\end{aligned}
$$

This overdetermined system, which we refer to as the JMMS equations, is in fact Frobenius integrable, and has a Hamiltonian structure which we summarize in the next subsection.

\section{1c. Hamiltonian Structure of the JMMS Equations}

For convenience, define a new normalization of the coordinates

$$
\begin{aligned}
q_{2 j} & :=-\frac{i}{2} x_{2 j}, \quad q_{2 j+1}:=\frac{1}{2} x_{2 j+1} \\
p_{2 j} & :=-i y_{2 j}, \quad p_{2 j+1}:=y_{2 j+1}
\end{aligned}
$$

and let

$$
G_{j}(x, y):=\frac{\pi^{2}}{4} x_{j}^{2}+y_{j}^{2}-\frac{1}{4} \sum_{\substack{k=1 \\ k \neq j}}^{2 m} \frac{\left(x_{j} y_{k}-x_{k} y_{j}\right)^{2}}{a_{j}-a_{k}}
$$

In terms of these quantities, we have

$$
\omega(a)=\sum_{j=1}^{2 m} G_{j}(x, y) d a_{j}
$$

Defining canonical Poisson brackets:

$$
\left\{x_{j}, x_{k}\right\}=\left\{y_{j}, y_{k}\right\}=0, \quad\left\{x_{j}, y_{k}\right\}=\delta_{j k}, \quad j, k=1, \ldots, 2 m
$$


the system of equations (1.24) is seen to have the nonautonomous Hamiltonian form

$$
d_{a} x_{j}=\left\{x_{j}, \omega(a)\right\} \quad \text { and } \quad d_{a} y_{j}=\left\{y_{j}, \omega(a)\right\}
$$

Moreover, the set of Hamiltonians $\left\{G_{j}\right\}_{j=1, \ldots 2 m}$ are easily verified to be generically functionally independent and in involution:

$$
\left\{G_{j}, G_{k}\right\}=0 \quad \text { for all } j, k=1, \ldots, 2 m \text {. }
$$

From this follows that the system (1.29), or equivalently (1.24), is Frobenius integrable, and that $\omega$ is locally an exact differential:

$$
\omega=d_{a} \log \tau
$$

where the value of the tau-function $\tau$ along the integral curves coincides with $\log (\operatorname{det}(I-\widehat{K}))$.

The significance of this Hamiltonian structure in terms of spectral invariants on loop algebras will be explained in $\S 2$. First, another similar system occurring in the computation of level spacing distribution functions of scaled random matrices at the "edge of the spectrum" will be described. For the details of what follows see [TW2]. (Here also only the case of equal $\lambda_{j}$ 's was considered, the generalization to the case of unequal $\lambda_{j}$ 's being straightforward.)

\section{1d. The Airy Kernel System and Distributions at the Edge of the Spectrum:}

Scaling at the "edge of the spectrum" [Mo], [Fo], [TW2], corresponds to choosing $X_{0} \sim \pm \sqrt{2 N}$ and gives rise to a different Fredholm determinant, in which the sine kernel (1.8) is replaced by the Airy kernel

$$
\begin{aligned}
K(x, y) & :=\frac{A(x) A^{\prime}(y)-A^{\prime}(x) A(y)}{x-y} \\
& =\lim _{N \rightarrow \infty} \frac{1}{2^{1 / 2} N^{1 / 6}} K_{N}\left(\sqrt{2 N}+\frac{x}{2^{1 / 2} N^{1 / 6}}, \sqrt{2 N}+\frac{y}{2^{1 / 2} N^{1 / 6}}\right)
\end{aligned}
$$

where now, $A(x)$ is an Airy function

$$
A(x)=\operatorname{Ai}(x)
$$

The logarithmic differential of the Fredholm determinant (1.14) in this case is also given by formula (1.21) where, using the same notation as above, the resolvent kernel $R(x, y)$ is still of the form $(1.19 \mathrm{a}, \mathrm{b})$, with the functions $Q(x, a), P(x, a)$ obtained by replacing $A(x)$ in 
eq. $(1.17 \mathrm{a}, \mathrm{b}),(1.18)$ by (1.33). Define $\left\{q_{j}, p_{j}\right\}_{j=1, \ldots 2 m}$ as in $(1.22 \mathrm{a}, \mathrm{b})$, and introduce two further quantities:

$$
\begin{aligned}
& u:=\sum_{j=1}^{m} \lambda_{j} \int_{a_{2 j-1}}^{a_{2 j}} A(x) Q(x, a) d x \\
& v:=\sum_{j=1}^{m} \lambda_{j} \int_{a_{2 j-1}}^{a_{2 j}} A(x) P(x, a) d x .
\end{aligned}
$$

Then similarly to the previous case, we have,

$$
\begin{aligned}
& R\left(a_{j}, a_{k}\right)=\frac{q_{j} p_{k}-p_{j} q_{k}}{a_{j}-a_{k}}, \quad j \neq k \\
& R\left(a_{j}, a_{j}\right)=\sum_{k \neq j}(-1)^{k} \frac{\left(q_{j} p_{k}-p_{j} q_{k}\right)^{2}}{a_{j}-a_{k}}+p_{j}^{2}-a_{j} q_{j}^{2}-2 p_{j} q_{j} u+2 q_{j}^{2} v,
\end{aligned}
$$

where all the limits are again taken within the intervals $J_{j}, J_{k}$. Differentiating with respect to the parameters $\left\{a_{j}\right\}_{j=1, \ldots 2 m}$ gives the system

$$
\begin{aligned}
\frac{\partial q_{j}}{\partial a_{k}} & =(-1)^{k} \frac{q_{j} p_{k}-p_{j} q_{k}}{a_{j}-a_{k}} q_{k} \quad(j \neq k), \\
\frac{\partial p_{j}}{\partial a_{k}} & =(-1)^{k} \frac{q_{j} p_{k}-p_{j} q_{k}}{a_{j}-a_{k}} p_{k} \quad(j \neq k), \\
\frac{\partial q_{j}}{\partial a_{j}} & =-\sum_{k \neq j}(-1)^{k} \frac{q_{j} p_{k}-p_{j} q_{k}}{a_{j}-a_{k}} q_{k}+p_{j}-q_{j} u \\
\frac{\partial p_{j}}{\partial a_{j}} & =-\sum_{k \neq j}(-1)^{k} \frac{q_{j} p_{k}-p_{j} q_{k}}{a_{j}-a_{k}} p_{k}+a_{j} q_{j}+p_{j} u-2 q_{j} v \\
\frac{\partial u}{\partial a_{j}} & =(-1)^{j} q_{j}^{2} \\
\frac{\partial v}{\partial a_{j}} & =(-1)^{j} p_{j} q_{j} .
\end{aligned}
$$

Again, introducing new coordinates $\left\{x_{j}, y_{j}\right\}_{j=0, \ldots 2 m}$ by:

$$
\begin{aligned}
q_{2 j} & :=-\frac{i}{2} x_{2 j}, & q_{2 j+1} & :=\frac{1}{2} x_{2 j+1} \\
p_{2 j} & :=-i y_{2 j}, & p_{2 j+1} & :=y_{2 j+1} \\
u & :=y_{0}, & v & :=\frac{1}{2} x_{0} .
\end{aligned}
$$

and defining canonical Poisson brackets

$$
\left\{x_{j}, x_{k}\right\}=\left\{y_{j}, y_{k}\right\}=0, \quad\left\{x_{j}, y_{k}\right\}=\delta_{j k}, \quad j, k=0, \ldots, 2 m
$$


eqs. (1.36) have the Hamiltonian form

$$
d_{a} x_{j}=\left\{x_{j}, \omega(a)\right\} \quad \text { and } \quad d_{a} y_{j}=\left\{y_{j}, \omega(a)\right\}, \quad j=0, \ldots, 2 m
$$

where

$$
\omega(a)=d_{a} \log (\operatorname{det}(I-\widehat{K}))=\sum_{j=1}^{2 m} G_{j}(x, y) d a_{j}
$$

and

$$
G_{j}(x, y)=y_{j}^{2}-\frac{1}{4} a_{j} x_{j}^{2}-x_{j} y_{j} y_{0}+\frac{1}{4} x_{j}^{2} x_{0}-\frac{1}{4} \sum_{\substack{k=1 \\ k \neq j}}^{2 m} \frac{\left(x_{j} y_{k}-x_{k} y_{j}\right)^{2}}{a_{j}-a_{k}} .
$$

It is straightforward to verify that these $G_{j}$ 's are also in involution, implying the Frobenius integrability of the system (1.40) and the existence of a $\tau$-function as in eq. (1.31). However, in this case, the phase space is $2(m+1)$-dimensional, so the $G_{j}$ 's alone do not form a complete set of commuting integrals. It is easily verified, though, that the following additional independent function

$$
G_{0}(x, y):=y_{0}^{2}-x_{0}-\frac{1}{4} \sum_{i=1}^{2 m} x_{i}^{2}
$$

is also in involution with the $G_{j}$ 's, thereby forming a complete set $\left\{G_{j}\right\}_{j=0, \ldots 2 m}$ of commuting integrals. Introducing an additional flow parameter $a_{0}$ corresponding to the Hamiltonian $G_{0}$, and letting

$$
\tilde{\omega}(\tilde{a}):=\omega(a)+G_{0} d a_{0},
$$

where

$$
\widetilde{a}:=\left(a_{0}, \ldots, a_{2 m}\right),
$$

we have the following extended system, which is also Frobenius integrable:

$$
d_{\tilde{a}} x_{j}=\left\{x_{j}, \tilde{\omega}(\tilde{a})\right\} \quad \text { and } \quad d_{\tilde{a}} y_{j}=\left\{y_{j}, \tilde{\omega}(\tilde{a})\right\}, \quad j=0, \ldots, 2 m
$$

Moreover, since $G_{0}$ is not explicitly dependent on the parameters $a_{j}$ and Poisson commutes with all the $\left\{G_{j}\right\}_{j=1, \ldots 2 m}$, it is in fact a constant. Since all the quantities $u, v, q_{j}, p_{j} \rightarrow 0$ as the $a_{j} \rightarrow \infty$, this constant value is just 0 .

\section{Classical $R$-Matrix and Isomonodromy Formulation}

Hamiltonian systems of the type encountered above can be understood within a general Lie algebraic setting (cf. $[\mathbf{A H P}],[\mathbf{H}],[\mathbf{R S}],[\mathbf{S}]$ ) which we now summarize. Let $\tilde{\mathbf{g}}$ be the 
loop algebra of smooth (real or complex), $s l(r)$-valued functions $X(\lambda)$, defined on a circle $S^{1}$ centered at the origin in the complex $\lambda$-plane. Define the Ad-invariant scalar product $<,>$ on $\tilde{\mathbf{g}}$ by:

$$
<X, Y>=\frac{1}{2 \pi i} \oint_{S^{1}} \operatorname{tr}(\mathrm{X}(\lambda) \mathrm{Y}(\lambda)) \mathrm{d} \lambda
$$

Interpreting this as the evaluation of a linear form $X$ on elements $Y \in \tilde{\mathbf{g}}$ allows us to identify $\tilde{\mathbf{g}}$ with an open, dense subspace of the dual space, which will be denoted $\tilde{\mathbf{g}}^{*}$. Under this identification, both the adjoint and coadjoint actions of the corresponding loop group $\tilde{\mathcal{G}}$ are given by conjugation:

$$
\operatorname{Ad}_{g}: X \longmapsto g X g^{-1}, \quad \operatorname{Ad}_{g}^{*}: X \longmapsto g X g^{-1}, \quad g \in \tilde{\mathcal{G}}, \quad X \in \tilde{\mathbf{g}} .
$$

As a linear space, $\tilde{\mathbf{g}}$ may be decomposed into a direct sum

$$
\tilde{\mathbf{g}}=\tilde{\mathbf{g}}_{+}+\tilde{\mathbf{g}}_{-},
$$

where $\tilde{\mathbf{g}}_{+}, \tilde{\mathbf{g}}_{-}$are the subalgebras consisting of elements $X(\lambda)$ that admit, respectively, holomorphic extensions to the interior and exterior of $S^{1}$, with the elements $X \in \tilde{\mathrm{g}}_{-}$satisfying $X(\infty)=0$. Under the identification $\tilde{\mathbf{g}} \sim \tilde{\mathbf{g}}^{*}$, we have

$$
\tilde{\mathbf{g}}_{+}^{*} \sim \tilde{\mathbf{g}}_{-}, \quad \tilde{\mathbf{g}}_{-}^{*} \sim \tilde{\mathbf{g}}_{+},
$$

where $\tilde{\mathbf{g}}_{ \pm}^{*} \subset \tilde{\mathbf{g}}^{*}$ are identified as the annihilators of $\tilde{\mathbf{g}}_{\mp}$.

Let

$$
\begin{gathered}
P_{ \pm}: \tilde{\mathbf{g}} \longrightarrow \tilde{\mathbf{g}}_{ \pm} \\
P_{ \pm}: X \longmapsto X_{ \pm}
\end{gathered}
$$

be the projections to these two subspaces relative to the decomposition (2.3) (determined, e.g., by splitting the Fourier series on $S^{1}$ into positive and negative parts) and define the endomorphism $R: \tilde{\mathbf{g}} \rightarrow \tilde{\mathbf{g}}$ as the difference:

$$
R:=P_{+}-P_{-} .
$$

Then $R$ is a classical $R$-matrix $[\mathbf{S}]$, in the sense that the bracket $[,]_{R}$ defined on $\tilde{\mathbf{g}}$ by:

$$
[X, Y]_{R}:=\frac{1}{2}[R X, Y]+\frac{1}{2}[X, R Y]
$$

is skew symmetric and satisfies the Jacobi identity, determining a new Lie algebra structure on the same space as $\tilde{\mathbf{g}}$, which we denote $\tilde{\mathbf{g}}_{R}$. The Lie Poisson bracket on $\tilde{\mathbf{g}}_{R}^{*} \sim \tilde{\mathbf{g}}^{*}$ associated to the Lie bracket $[,]_{R}$ is:

$$
\left.\{f, g\}\right|_{X}=<[d f, d g]_{R}, X>
$$


for smooth functions $f, g$ on $\tilde{\mathbf{g}}_{R}^{*}$ (with the usual identifications, $\left.d f\right|_{X},\left.d g\right|_{X} \in \tilde{\mathbf{g}}^{*} \sim \tilde{\mathbf{g}}$ ). The corresponding group $\tilde{\mathcal{G}}_{R}$ is identified with the direct product $\tilde{\mathcal{G}}_{+} \times \tilde{\mathcal{G}}_{-}$, where $\tilde{\mathcal{G}}_{ \pm}$are the loop groups associated to $\tilde{\mathbf{g}}_{ \pm}$. The adjoint and coadjoint actions are given by:

$$
\begin{aligned}
& \operatorname{Ad}_{R}(g):\left(X_{+}+X_{-}\right) \longmapsto g_{+} X_{+} g_{+}^{-1}+g_{-} X_{-} g_{-}^{-1} \\
& \operatorname{Ad}_{R}^{*}(g):\left(X_{+}+X_{-}\right) \longmapsto\left(g_{-} X_{+} g_{-}^{-1}\right)_{+}+\left(g_{+} X_{-} g_{+}^{-1}\right)_{-}, \\
& X_{ \pm} \in \tilde{\mathrm{g}}_{ \pm}, \quad g_{ \pm} \in \tilde{\mathcal{G}}_{ \pm}
\end{aligned}
$$

Let $a:=\left\{a_{i}\right\}_{i=1}^{n}$ be a set of $n$ distinct real (or complex) numbers and $\kappa:=\left\{k, k_{i}\right\}_{i=1}^{n}$ a set of $n+1$ nonnegative integers. Define the subspace $\mathbf{g}_{a, \kappa} \subset \tilde{\mathbf{g}}_{R}^{*}$ to consist of elements $X=X_{+}+X_{-}$with $X_{+} \in \tilde{\mathbf{g}}_{+}$a polynomial in $\lambda$ of degree $k$ and $X_{-} \in \tilde{\mathbf{g}}_{-}$a rational function of $\lambda$, with poles of degree $\left\{k_{1}, \ldots, k_{n}\right\}$ at the points $\left\{a_{1}, \ldots, a_{n}\right\}$. Then $\mathbf{g}_{a, \kappa} \subset \tilde{\mathbf{g}}_{R}^{*}$ is $A d_{R^{-}}^{*}$ invariant, and hence a Poisson subspace. The coefficient $Y$ of the leading term in the degree $k$ polynomial $X_{+}$is $A d_{R}^{*}$ invariant, and hence constant under all Hamiltonian flows. Let $\mathcal{I}_{a, \kappa}:=\left.I\left(\tilde{\mathbf{g}}^{*}\right)\right|_{\mathbf{g}_{a, \kappa}}$ be the ring of $A d^{*}$-invariant polynomials on $\tilde{\mathbf{g}}^{*} \sim \tilde{\mathbf{g}}_{R}^{*}$, restricted to $\mathbf{g}_{a, \kappa}$, and define, for $X \in \mathbf{g}_{a, \kappa}$, the $s l(r)$-valued polynomial function:

$$
L(\lambda):=a(\lambda) X(\lambda),
$$

where

$$
a(\lambda):=\prod_{i=1}^{n}\left(\lambda-a_{i}\right)^{k_{i}} .
$$

Then the ring $\mathcal{I}_{a, \kappa}$ is generated by the coefficients of the characteristic polynomial:

$$
\mathcal{P}(\lambda, z):=\operatorname{det}(L(\lambda)-z I),
$$

and the characteristic equation

$$
\mathcal{P}(\lambda, z)=0
$$

defines, after suitable compactification (and possible desingularization), an algebraic curve $\Gamma$, called the "spectral curve", which is generically an $r$-fold branched cover of $\mathbf{C P}^{1}$.

It follows from the Adler-Kostant-Symes (AKS) theorem that:

1) The ring $\mathcal{I}_{a, \kappa}$ is Poisson commutative (with respect to the $\{,\}_{R}$ Poisson bracket).

2) For a Hamiltonian $G \in \mathcal{I}_{a, \kappa}$, Hamilton's equations are given by:

$$
\frac{d X}{d t}=\{X, G\}=\left[d G_{+}, X\right]=-\left[d G_{-}, X\right]
$$


(The last equality following from the fact that $[d G, X]=0$, which is the infinitesimal form of $\mathrm{Ad}^{*}$-invariance).

This means that the spectral curve $\Gamma$ is invariant under the flows generated by the elements of $\mathcal{I}_{a, \kappa}$, since any two coefficients of the spectral polynomial $\mathcal{P}(\lambda, z)$ Poisson commute. It may also be shown $[\mathbf{A H P}],[\mathbf{A H H 1}]$ that, for sufficiently generic initial conditions and coadjoint orbits $\mathcal{O}$ in $\mathbf{g}_{a, \kappa}$, the elements of $\mathcal{I}_{a, \kappa}$, restricted to $\mathcal{O} \subset \mathbf{g}_{a, \kappa}$, define completely integrable Hamiltonian systems; that is, the number of independent generators in $\mathcal{I}_{a, \kappa}$ equals one half the dimension of the orbit. Through a standard construction [AvM], [RS], [AHH1], [AHH2], the flows they generate are shown to linearize on the Jacobi variety associated to $\Gamma$.

We now specialize to the case $\left\{k=1, k_{i}=1\right\}_{i=1, \ldots n}$. Then $X(\lambda)$ has the form

$$
X(\lambda)=\lambda Y+N_{0}+\sum_{i=1}^{n} \frac{N_{i}}{\lambda-a_{i}}
$$

and the $\operatorname{Ad}_{R}^{*}$-action is:

$$
A d_{R}^{*}\left(g_{+}, g_{-}\right): X(\lambda) \longmapsto \lambda Y+N_{0}+\left[\gamma_{0}, Y\right]+\sum_{i=1}^{n} \frac{g_{i} N_{i} g_{i}^{-1}}{\lambda-a_{i}}
$$

where

$$
\gamma_{0}:=\frac{1}{2 \pi i} \oint_{S^{1}} g_{-}(\lambda) d \lambda, \quad g_{i}:=g_{+}\left(a_{i}\right), \quad i=1, \ldots n .
$$

Hence, each coadjoint orbit in $\mathbf{g}_{a, \kappa}$ consists of elements of the form:

$$
X(\lambda)=\lambda Y+C+\left[\gamma_{0}, Y\right]+\sum_{i=1}^{n} \frac{N_{i}}{\lambda-a_{i}},
$$

where

$$
N_{0}:=C+\left[\gamma_{0}, Y\right],
$$

with $Y$ and $C$ constant matrices, and the $N_{i}$ 's have fixed Jordan normal form.

Let

$$
\frac{1}{2} \operatorname{tr} X^{2}=y \lambda^{2}+c \lambda+G_{0}+\sum_{i=1}^{n} \frac{G_{i}}{\lambda-a_{i}}+\sum_{i=1}^{n} \frac{K_{i}}{\left(\lambda-a_{i}\right)^{2}},
$$

where

$$
y:=\frac{1}{2} \operatorname{tr} Y^{2}, \quad c:=\operatorname{tr}\left(Y N_{0}\right)=\operatorname{tr}(Y C)
$$

and 


$$
\begin{aligned}
G_{0} & :=\frac{1}{4 \pi i} \oint_{S^{1}} \operatorname{tr} X^{2}(\lambda) \frac{d \lambda}{\lambda}=\operatorname{tr}\left(Y \sum_{i=1}^{n} N_{i}\right)+\frac{1}{2} \operatorname{tr} N_{0}^{2} \\
G_{i} & :=\frac{1}{4 \pi i} \oint_{\lambda=a_{i}} \operatorname{tr} X^{2}(\lambda) d \lambda=a_{i} \operatorname{tr}\left(Y N_{i}\right)+\operatorname{tr}\left(N_{0} N_{i}\right)+\sum_{j \neq i}^{n} \frac{\operatorname{tr}\left(N_{i} N_{j}\right)}{a_{i}-a_{j}} \\
K_{i} & :=\frac{1}{4 \pi i} \oint_{\lambda=a_{i}}\left(\lambda-a_{i}\right) \operatorname{tr} X^{2}(\lambda) d \lambda=\frac{1}{2} \operatorname{tr}\left(N_{i}^{2}\right) \\
i & =1, \ldots n
\end{aligned}
$$

with the integrals $\oint_{\lambda=a_{i}}$ taken around a small circle enclosing only this pole. Then $y, c$ and $\left\{K_{i}\right\}_{i=1, \ldots n}$ are all Casimir invariants and hence constant on each $\operatorname{Ad}_{R}^{*}$ orbit, and $\left\{G_{i}\right\}_{i=0, \ldots n}$ are independent elements of the Poisson commuting ring $\mathcal{I}_{a, \kappa}$. Denoting the time parameters for the Hamiltonian flows generated by $\left\{G_{i}\right\}_{i=0, \ldots n}$ as $\left\{t_{i}\right\}_{i=0, \ldots n}$, the AKS theorem implies that Hamilton's equations are of the form:

$$
\begin{aligned}
& \frac{\partial X}{\partial t_{0}}=\left\{X, G_{0}\right\}=\left[\left(d G_{0}\right)_{+}, X\right] \\
& \frac{\partial X}{\partial t_{i}}=\left\{X, G_{i}\right\}=-\left[\left(d G_{i}\right)_{-}, X\right], \quad i=1, \ldots n
\end{aligned}
$$

where

$$
\left(d G_{0}\right)_{+}=Y, \quad\left(d G_{i}\right)_{-}=-\frac{N_{i}}{\lambda-a_{i}}, \quad i=1, \ldots n
$$

Evaluating residues at $\left\{\lambda=a_{i}\right\}_{i=1, \ldots n}$ and $\lambda=\infty$, we see that this is equivalent to:

$$
\begin{array}{rlrl}
\frac{\partial N_{i}}{\partial t_{0}} & =\left[Y, N_{i}\right], & \frac{\partial N_{0}}{\partial t_{0}}=\left[Y, N_{0}\right], & \frac{\partial N_{0}}{\partial t_{i}}=\left[Y, N_{i}\right], \\
\frac{\partial N_{i}}{\partial t_{j}} & =\frac{\left[N_{i}, N_{j}\right]}{a_{i}-a_{j}}, \quad i \neq j, & \quad \frac{\partial N_{i}}{\partial t_{i}}=\left[a_{i} Y+N_{0}+\sum_{j \neq i} \frac{N_{j}}{a_{i}-a_{j}}, N_{i}\right] \\
i, j & =1, \ldots n .
\end{array}
$$

If we now reinterpret these as nonautonomous Hamiltonian systems, by identifying the constants $\left\{a_{i}\right\}$ with the flow parameters $\left\{t_{i}=a_{i}\right\}_{i=1, \ldots n}$, system (2.22) becomes (cf. [JMMS], [JMU]):

$$
\begin{array}{rlrl}
\frac{\partial N_{i}}{\partial a_{0}} & =\left[Y, N_{i}\right], & \frac{\partial N_{0}}{\partial a_{0}}=\left[Y, N_{0}\right], & \frac{\partial N_{0}}{\partial a_{i}}=\left[Y, N_{i}\right], \\
\frac{\partial N_{i}}{\partial a_{j}} & =\frac{\left[N_{i}, N_{j}\right]}{a_{i}-a_{j}}, & i \neq j, & \frac{\partial N_{i}}{\partial a_{i}}=\left[a_{i} Y+N_{0}+\sum_{j \neq i} \frac{N_{j}}{a_{i}-a_{j}}, N_{i}\right] \\
i, j & =1, \ldots n .
\end{array}
$$


(Here $t_{0}$ has similarly been renamed $a_{0}$, but it does not appear as a parameter in the system, and hence represents autonomous flow.) This system is equivalent to the commutativity of the following system of differential operators:

$$
\begin{aligned}
\mathcal{D}_{\lambda} & :=\frac{\partial}{\partial \lambda}-\lambda Y-N_{0}-\sum_{i=1}^{n} \frac{N_{i}}{\lambda-a_{i}}, \\
\mathcal{D}_{i} & :=\frac{\partial}{\partial a_{i}}+\frac{N_{i}}{\lambda-a_{i}}, \\
\mathcal{D}_{0} & :=\frac{\partial}{\partial a_{0}}-Y .
\end{aligned}
$$

It follows that the monodromy of the operator $\mathcal{D}_{\lambda}$ is preserved under the deformations parametrized by $\left\{a_{0}, a_{1}, \ldots, a_{n}\right\}$.

To obtain the system (1.26)-(1.29), we take the $\left\{a_{j}\right\}_{j=1}^{n}$ as real, $r=2, n=2 m, Y=0$ (so really, we are in the Poisson subspace with $k=0$ ), and choose

$$
C=\left(\begin{array}{cc}
0 & \frac{\pi^{2}}{2} \\
-2 & 0
\end{array}\right)
$$

and the $N_{i}$ 's with Jordan normal form $\left(\begin{array}{ll}0 & 0 \\ 1 & 0\end{array}\right)$. The $\operatorname{Ad}_{R}^{*}$ orbit $\mathcal{O}_{0} \subset \mathbf{g}_{a, \kappa}$ then consists of elements of the form:

$$
X(\lambda)=\left(\begin{array}{cc}
0 & \frac{\pi^{2}}{2} \\
-2 & 0
\end{array}\right)+\frac{1}{2} \sum_{i=1}^{2 m} \frac{\left(\begin{array}{cc}
-x_{i} y_{i} & -y_{i}^{2} \\
x_{i}^{2} & x_{i} y_{i}
\end{array}\right)}{\lambda-a_{i}} .
$$

Here, we have parametrized the orbit by elements of $M_{0}:=\mathbf{R}^{4 m} /\left(\mathbf{Z}_{2}\right)^{4 m}$, where $\left(\left(x_{1}, \ldots, x_{2 m}\right),\left(y_{1}, \ldots, y_{2 m}\right)\right) \in \mathbf{R}^{4 m}$, and the $\left(\mathbf{Z}_{2}\right)^{4 m}$ action is generated by reflections in the coordinate hyperplanes:

$$
\begin{gathered}
\left(\epsilon_{1}, \ldots, \epsilon_{2 m}\right):\left(\left(x_{1}, \ldots, x_{2 m}\right),\left(y_{1}, \ldots, y_{2 m}\right)\right) \\
\longmapsto\left(\left(\epsilon_{1} x_{1}, \ldots, \epsilon_{2 m} x_{2 m}\right),\left(\epsilon_{1} y_{1}, \ldots, \epsilon_{2 m} y_{2 m}\right)\right) \\
\left\{\epsilon_{i}= \pm 1\right\}_{i=1, \ldots 2 m} .
\end{gathered}
$$

The map $J_{0}: M_{0} \longrightarrow \tilde{\mathbf{g}}_{R}^{*}$ defined by

$$
J_{0}:\left(\left(x_{1}, \ldots, x_{2 m}\right),\left(y_{1}, \ldots, y_{2 m}\right) \longmapsto\left(\begin{array}{cc}
0 & \frac{\pi^{2}}{2} \\
-2 & 0
\end{array}\right)+\frac{1}{2} \sum_{i=1}^{2 m} \frac{\left(\begin{array}{cc}
-x_{i} y_{i} & -y_{i}^{2} \\
x_{i}^{2} & x_{i} y_{i}
\end{array}\right)}{\lambda-a_{i}}\right.
$$

is a symplectic embedding with respect to the standard symplectic structure:

$$
\omega=\sum_{i=1}^{2 m} d x_{i} \wedge d y_{i}
$$


on $M_{0}$ and the orbital (Lie-Kostant-Kirillov) symplectic structure on $\mathcal{O}_{0}$. (This is a special case of the moment map embeddings into rational Poisson subspaces of the dual of loop algebras developed in [AHP], [AHH3].) Evaluating the Hamiltonians $\left\{G_{i}\right\}_{i=1, \ldots 2 m}$ defined in (2.20) for this case gives those of eqs. (1.26) and the $\left\{a_{j}\right\}, j \neq 0$ components of the equations of motion (2.24) are equivalent to (1.29), up to quotienting by the $\left(\mathbf{Z}_{2}\right)^{4 m}$ action (2.28).

To obtain the system (1.40)-(1.45), we again take $r=2, n=2 m$, but choose

$$
Y=\left(\begin{array}{cc}
0 & -\frac{1}{2} \\
0 & 0
\end{array}\right), \quad C=\left(\begin{array}{cc}
0 & 0 \\
-2 & 0
\end{array}\right), \quad\left[\gamma_{0}, Y\right]=\left(\begin{array}{cc}
y_{0} & \frac{x_{0}}{2} \\
0 & -y_{0}
\end{array}\right)
$$

with the $N_{i}$ 's again of Jordan normal form $\left(\begin{array}{ll}0 & 0 \\ 1 & 0\end{array}\right)$. In this case, the $\operatorname{Ad}_{R}^{*}$ orbit $\mathcal{O}_{1} \subset \mathbf{g}_{a, \kappa}$ consists of elements of the form:

$$
X(\lambda)=\left(\begin{array}{cc}
y_{0} & -\frac{\lambda}{2}+\frac{x_{0}}{2} \\
-2 & -y_{0}
\end{array}\right)+\frac{1}{2} \sum_{i=1}^{2 m} \frac{\left(\begin{array}{cc}
-x_{i} y_{i} & -y_{i}^{2} \\
x_{i}^{2} & x_{i} y_{i}
\end{array}\right)}{\lambda-a_{i}} .
$$

Here, we have parametrized the orbit by elements of $M_{1}:=\mathbf{R}^{2} \times \mathbf{R}^{4 m} /\left(\mathbf{Z}_{2}\right)^{4 m}$, where $\left(x_{0}, y_{0}\right) \in$ $\mathbf{R}^{2},\left(\left(x_{1}, \ldots, x_{2 m}\right),\left(y_{1}, \ldots, y_{2 m}\right)\right) \in \mathbf{R}^{4 m}$, and the $\left(\mathbf{Z}_{2}\right)^{4 m}$ action is again given by (2.28). The map $J_{1}: M_{1} \longrightarrow \tilde{\mathbf{g}}_{R}^{*}$ defined by

$$
J_{1}:\left(\left(x_{0}, x_{1}, \ldots, x_{2 m}\right),\left(y_{0}, y_{1}, \ldots, y_{2 m}\right) \longmapsto\left(\begin{array}{cc}
y_{0} & -\frac{\lambda}{2}+\frac{x_{0}}{2} \\
-2 & -y_{0}
\end{array}\right)+\frac{1}{2} \sum_{i=1}^{2 m} \frac{\left(\begin{array}{cc}
-x_{i} y_{i} & -y_{i}^{2} \\
x_{i}^{2} & x_{i} y_{i}
\end{array}\right)}{\lambda-a_{i}}\right.
$$

is again a symplectic embedding, now with respect to the symplectic structure:

$$
\omega=d x_{0} \wedge d y_{0}+\sum_{i=1}^{2 m} d x_{i} \wedge d y_{i}
$$

on $M_{1}$ and the orbital (Lie-Kostant-Kirillov) symplectic structure on $\mathcal{O}_{1}$. Evaluating the Hamiltonians $\left\{G_{i}\right\}_{i=0, \ldots 2 m}$ defined in (2.20) for this case gives those of eqs. (1.41), (1.42) and the equations of motion (2.24) are equivalent to (1.45) - again, up to quotienting by the $\left(\mathbf{Z}_{2}\right)^{4 m}$ action $(2.28)$.

For this case, eqs. (2.19), (2.31), (2.33) give $y=0, c=1$ and $\operatorname{tr}\left(N_{i}^{2}\right)=0$, so

$$
-\operatorname{det}(X)=\frac{1}{2} \operatorname{tr}\left(X^{2}\right)=\lambda+G_{0}+\sum_{i=1}^{2 m} \frac{G_{i}}{\lambda-a_{i}}:=\frac{P(\lambda)}{a(\lambda)}
$$


where $P(\lambda)$ is a monic polynomial of degree $2 m+1$

$$
P(\lambda)=\lambda^{2 m+1}+P_{2 m} \lambda^{2 m}+\ldots+P_{0}
$$

with

$$
P_{2 m}=G_{0}-\sum_{i=1}^{2 m} a_{i}, \quad P_{2 m-1}=\sum_{i=1}^{2 m} G_{i}-G_{0} \sum_{i=1}^{2 m} a_{i}, \quad \text { etc. }
$$

From eqs. (2.9)-(2.11), we have the following equation for the spectral curve $\Gamma$ :

$$
\mathcal{P}(\lambda, z)=z^{2}-a(\lambda) P(\lambda)=0
$$

which shows that it is hyperelliptic, of genus $g=2 m$, with $2 m+1$ of its branch points located at $\left\{a_{1}, \ldots a_{2 m}, \infty\right\}$ and the others determined by the values of the roots of $P(\lambda)$. For the system (1.26)-(1.29) associated to the sine kernel, the leading term in (2.18) vanishes, so the polynomial $P(\lambda)$ is of degree $2 m$ and the curve has genus $g=2 m-1$, with no branch points at $\infty$. For the autonomous system (2.23), these curves are invariant under the flows. For the nonautonomous system (2.24), which in these cases reduces to (1.29) or (1.45), it would be of interest to determine the dependence of the spectral curves on the deformation parameters $\left\{a_{i}\right\}_{i=0, \ldots 2 m}$ through a system of PDE's involving the spectral invariants $\left\{G_{i}\right\}_{i=0, \ldots 2 m}$ alone. For the single finite interval case, this is given in [JMMS], [TW1] by the $\tau$-function form of the Painlevé equation $P_{V}$, while for the single semi-infinite interval case, this is given in [TW2] by the corresponding $\tau$-function form of $P_{I I}$.

\section{Acknowledgments}

It is a pleasure to acknowledge E. L. Basor, F. J. Dyson, P. J. Forrester, A. Its, and M. L. Mehta for their helpful comments and encouragement and for sending us their preprints prior to publication. The second author wishes to thank the organizers of the program "Low Dimensional Topology and Quantum Field Theory" for their kind hospitality at the Isaac Newton Institute for Mathematical Sciences. Particular thanks to R. J. Baxter and H. Osborn for making the stay at Cambridge most pleasant. 


\section{References}

[AHH1] Adams, M. R., Harnad, J. and Hurtubise, J., "Isospectral Hamiltonian Flows in Finite and Infinite Dimensions II. Integration of Flows," Commun. Math. Phys. 134, 555-585 (1990).

[AHH2] Adams, M. R., Harnad, J. and Hurtubise, J., "Darboux Coordinates and Liouville-Arnold Integration in Loop Algebras," preprint CRM (1992) (to appear in Commun. Math. Phys. (1993)) .

[AHH3] Adams, M. R., Harnad, J. and Hurtubise, J., "Dual Moment Maps to Loop Algebras," Lett. Math. Phys. 20, 294-308 (1990).

[AHP] Adams, M. R., Harnad, J. and Previato, E., "Isospectral Hamiltonian Flows in Finite and Infinite Dimensions I. Generalised Moser Systems and Moment Maps into Loop Algebras," Commun. Math. Phys. 117, 451-500 (1988).

[AvM] Adler, M. and van Moerbeke, P., "Completely Integrable Systems, Euclidean Lie Algebras, and Curves," Adv. Math. 38, 267-317 (1980); "Linearization of Hamiltonian Systems, Jacobi Varieties and Representation Theory," ibid. 38, 318-379 (1980).

[BTW] Basor, E. L., Tracy, C. A., and Widom, H., "Asymptotics of Level-Spacing Distributions for Random Matrices," Phys. Rev. Letts. 69, 5-8 (1992).

[Dy] Dyson, F. J., "The Coulomb Fluid and the Fifth Painlevé Transcendent," IASSNSS-HEP-92/43 preprint, to appear in the proceedings of a conference in honor of C. N. Yang, ed. S.-T. Yau.

[Fo] Forrester, P. J., "The Spectrum Edge of Random Matrix Ensembles," preprint.

[H] Harnad, J., "Dual Isomonodromy Deformations and Moment Maps to Loop Algebras," preprint CRM1844 (1993).

[IIKS] Its, A. R., Izergin, A. G., Korepin, V. E. and Slavnov, N. A., "Differential Equations for Quantum Correlation Functions," Int. J. Mod. Phys. B4, 1003-1037 (1990).

[IIKV] Its, A. R., Izergin, A. G., Korepin, V. E. and Varzugin, G. G., "Large Time and Distance Asymptotics of Field Correlation Function of Impenetrable Bosons at Finite Temperature," Physica 54D, 351-395 (1992).

[JMMS] Jimbo, M., Miwa, T., Môri, Y. and Sato, M.,, "Density Matrix of an Impenetrable Bose Gas and the Fifth Painlevé Transcendent", Physica 1D, 80-158 (1980).

[JMU] Jimbo, M., Miwa, T., and Ueno, K., "Monodromy Preserving Deformation of Linear Ordinary Differential Equations with Rational Coeefficients I.," Physica 2D, 306-352 (1981).

[JM] Jimbo, M., and Miwa, T., "Monodromy Preserving Deformation of Linear Ordinary Differential Equations with Rational Coeefficients II, III.," Physica 2D, 407-448 (1981); ibid., 4D, 26-46 (1981).

[Mc] McCoy, B. M., "Spin Systems, Statistical Mechanics and Painlevé Functions," in Painlevé Transcendents: Their Asymptotics and Physical Applications, eds. D. Levi and P. Winternitz, Plenum Press, New York (1992), pgs. 377-391.

[Me1] Mehta, M. L., Random Matrices, 2nd ed., Academic Press, San Diego, (1991).

[Me2] Mehta, M. L., "A Non-linear Differential Equation and a Fredholm Determinant," J. de Phys. I2,17211729 (1992).

[MM] Mehta, M. L. and Mahoux, G., "Level Spacing Functions and Non-linear Differential Equations," SPh$\mathrm{T} / 92-107$ preprint.

[Mo] Moore, G., "Matrix Models of 2D Gravity and Isomonodromic Deformation," Prog. Theor. Phys. Suppl. No. 102, 255-285 (1990).

[MTW] McCoy, B. M., Tracy, C. A., and Wu, T. T., "Painlevé Functions of the Third Kind," J. Math. Phys. 18, 1058-1092 (1977). 
[Po] Porter, C. E., Statistical Theory of Spectra: Fluctuations, Academic Press, New York (1965).

[RS] Reiman, A. G., and Semenov-Tian-Shansky, M.A., "Reduction of Hamiltonian systems, Affine Lie algebras and Lax Equations I, II," Invent. Math. 54, 81-100 (1979); ibid. 63, 423-432 (1981).

[S] Semenov-Tian-Shansky, M. A., "What is a Classical R-Matrix," Funct. Anal. Appl. 17 259-272 (1983); "Dressing Transformations and Poisson Group Actions," Publ. RIMS Kyoto Univ. 21 1237-1260 (1985).

[SMJ] Sato, M., Miwa, T., and Jimbo, M., "Holonomic Quantum Fields I-V," Publ. RIMS Kyoto Univ. 14, 223-267 (1978); ibid. 15, 201-278 (1979); ibid. 15, 577-629 (1979); ibid.15, 871-972 (1979); ibid. 16, 531-584 (1980).

[TW1] Tracy, C. A., and Widom, H., "Introduction to Random Matrices," UCD preprint ITD 92/93-10 (1992), to appear in VIIIth Scheveningen Conf. Proc., Springer Lecture Notes in Physics.

[TW2] Tracy, C. A., and Widom, H., "Level Spacing Distributions and the Airy Kernel," UCD preprint ITD 92/93-9 (1992).

[W] Widom, H., "The Asymptotics of a Continuous Analogue of Orthogonal Polynomials," to appear in J. Approx. Th.

[WMTB] Wu, T. T., McCoy, B. M., Tracy, C. A., and Barouch, E., "Spin-Spin Correlation Functions for the Two-Dimensional Ising Model: Exact Theory in the Scaling Region," Phys. Rev. B13, 316-374 (1976). 